\title{
Micropapillary Adenocarcinoma of the Lung that Metastasized to the Same Site in the Brain Twice: A Rare Case Report and Review of Literature
}

\author{
Ahmad Faried ${ }^{1,2 *}$, Rhonaz P. Agung ${ }^{1}$, Hasrayati Agustina ${ }^{2,3}$, Bethy S. Hernowo ${ }^{3}$, Muhammad Z. Arifin ${ }^{1}$ \\ ${ }^{1}$ Department of Neurosurgery, Faculty of Medicine, Universitas Padjadjaran (FK UNPAD), Dr. Hasan Sadikin Hospital (RSHS), \\ Bandung, Indonesia; ${ }^{2}$ Department of Oncology and Stem Cell Working Group, Faculty of Medicine, Universitas Padjadjaran \\ (FK UNPAD), Dr. Hasan Sadikin Hospital (RSHS), Bandung, Indonesia; ${ }^{3}$ Department of Anatomical Pathology, FK UNPAD, \\ RSHS, Bandung, Indonesia
}

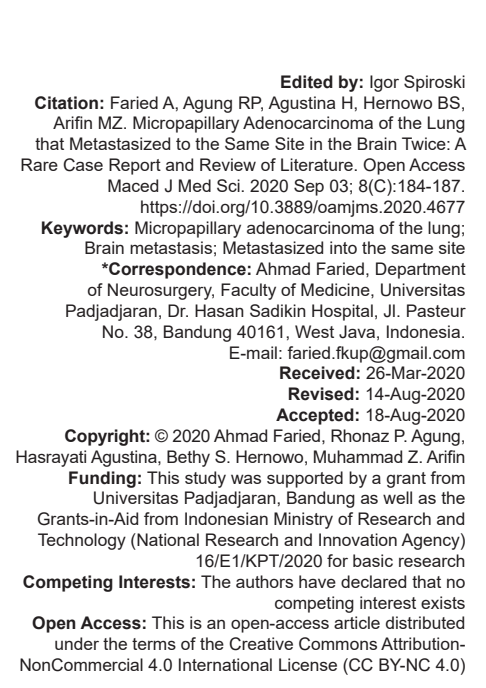

\begin{abstract}
BACKGROUND: Adenocarcinoma (ADC) of the lung is the most common subtype of non-small cell lung cancer (NSCLC), occurring in $\sim 40 \%$ of all patients with significantly higher incidence of brain metastasis compared with other subtypes of NSCLC. Among the five subtypes ADC of the lung, micropapillary predominant ADC may be more likely to metastasize. There are almost no reports of micropapillary ADC of the lung initially discovered to metastasis into the same site in the brain that has been previously operated.

CASE REPORT: We reported a 54-year-old woman who was referred to ICU of Dr. Hasan Sadikin Hospital, Bandung due to a decreased of consciousness. Head computed tomography scan revealed multiple isohypodense lesions in the right frontal with brain edema. She had craniotomy total tumor removal 8 months earlier and diagnosed as brain metastasis due to micropapillary ADC of the lung. She refused to receive chemoradiotherapy. At the operation site, multilobulated lesions were found, and hence, she was operated for the $2^{\text {nd }}$ time at the same site with the first one exhibited the same histology. Immunohistological shown positive results for thyroid transcription factor (TTF)-1 and cytokeratin (CK)-7; negative for CK-20, led to a diagnosis of micropapillary ADC of the lung.

CONCLUSION: Herein, we reported our experience regarding a case of micropapillary ADC of the lung, considered as poorly differentiated ADC and associated with a high-grade lesion that metastasized to the same site that had been previously operated, twice. A definitive diagnosis was possible only through a histopathological examination along with a good communication between the surgeon and the pathologist.
\end{abstract}

\section{Introduction}

Adenocarcinoma (ADC) of the lung is the most common subtype of non-small cell lung cancer (NSCLC), occurring in $\sim 40 \%$ of all patients, is characterized by rapid progression or higher incidence of early brain metastasis compared with other subtypes of NSCLC [1]. The International Association for the Study of Lung Cancer/ American Thoracic Society/European Respiratory Society criteria for lung ADC classification [2] and the 2015 WHO criteria lung tumor classification recommend the identification of pathological patterns [3]. Primary invasive lung ADC has five architectural growth patterns, including lepidic, acinar, papillary, solid, and micropapillary patterns. Tsuta et al. reported that the most prevalent subtypes of invasive lung ADC was papillarypredominant $\quad(37.1 \%), \quad$ lepidicpredominant $(15.1 \%)$, solid tumor with mucin production predominant (13.7\%), acinar predominant (10.8\%), and micropapillary predominant $(6.7 \%)$ [4]. Herein, we reported our experience regarding a case of micropapillary ADC of the lung, considered as poorly differentiated $A D C$, and associated with a high-grade metastasized lesions to the same site that had been previously operated, twice; the first surgery had been performed 8 months before at the very same area in the brain, the right frontal lobe.

\section{Case Presentation}

We present the case of a 54-year-old woman who suffered from intermittent headaches that worsened overtime but could be relieved with analgesics along with behavioral changes over the preceding 2 months. Neurological examinations and other cranial nerves revealed normal findings with no motor and sensory deficits. Chest radiograph (Figure 1a) and blood examination results were also within normal limits. Computed tomography (CT) scan of the head revealed 
an irregular isohypodense lesion in the right frontal region that was enhanced inhomogeneously with contrast, ring enhancement (+), peritumoral edema $(+)$, midline shift $>5 \mathrm{~mm}$ to the left, and sulcus and gyrus compressed (Figure 1b) and a pre-operative diagnosis of high-grade glioma was made.
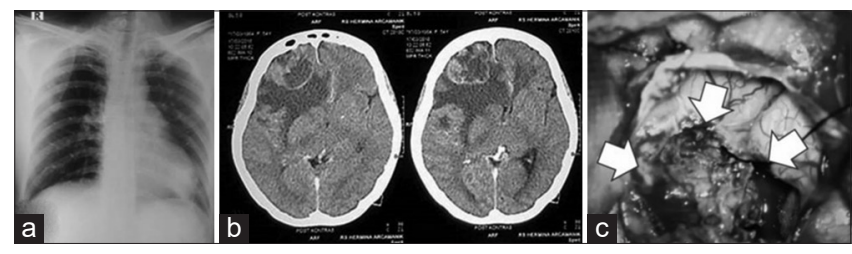

Figure 1: A pre-operative chest radiograph (a). The head computed tomography scan with contrast showing a large right frontal irregular isohypodense lesion with ring enhancement central necrosis appearance (b). Tumor was grayish, not easy on suction, highly vascular, easy to be bleed (c)

A craniotomy tumor removal surgery in the supine position using a right pterional approach was performed. The tumor was found to be grayish, not easy to suction, highly vascular, bleeding easily, and sticking to the meninges and measured $7 \times 5 \mathrm{~cm}$ (Figure 1c). Histological examination confirmed the presence of micropapillary patterns predominant and perivascular arrangement (Figure 2a). The cells were strongly diffuse and demonstrated positive immunoreactivity to cytokeratin (CK) and moderately positive immunoreactivity to epithelial membrane antigen (EMA) (Figure 2b and c). Immunoreactivity to glial fibrillary acidic protein (GFAP) and vimentin was negative (Figure $2 \mathrm{~d}$ and e). Tumor cells had round nuclei with a regular shape, chromatin, and a moderate amount of cytoplasm with distinct cell borders; hence, they contained prominent nuclei. The Ki-67 rate of labeling index was $40 \%$ (Figure $2 f$ ). Finally, the tumor was diagnosed as micropapillary ADC of the lung based on histological and immunohistochemical (IHC) findings; thyroid transcription factor-1 (TTF-1) and CK-7 positive, CK-20 negative (data not shown). Diagnose of lung carcinoma metastasis was made even the chest radiograph within normal limit (Figure 1a). Post-operative chemoradiotherapy was planned, but the patient refused.

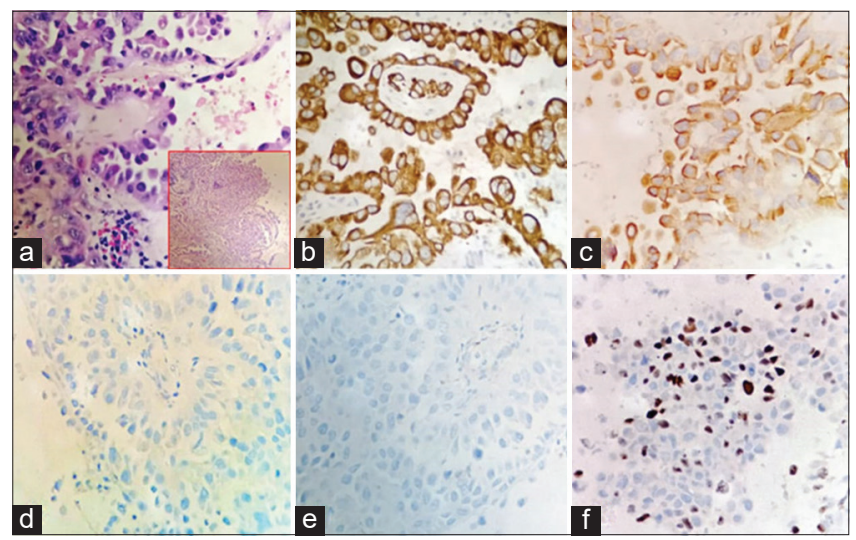

Figure 2: Photomicrograph showing the sheets of the tumor cells arrange in papillary and perivascular pseudopapillary patterns (a) ( $H$ and $E, \times 400$; inset $H$ and $E \times 100)$. The immunohistochemical profile shows positivity for cytokeratin (b) and epithelial membrane antigen (c); negativity for GFAP (d) and vimentin (e) (×400). Tumor cells positivity for proliferative marker Ki-67 (f) (×400)

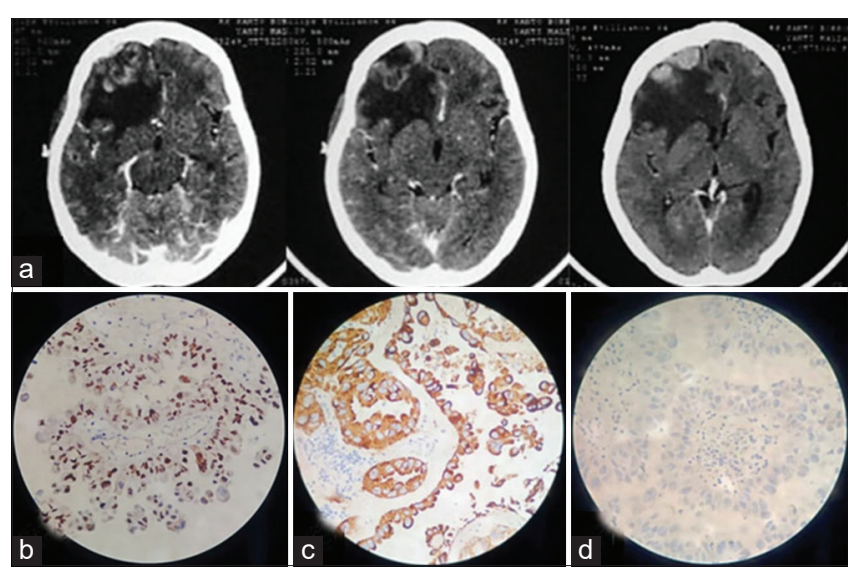

Figure 3: Following a recently head computed tomography scan showed the presence of multilobulated lesion at the very same site of previously performed total tumor removal (a). Immunohistochemical positivity for thyroid transcription factor-1 (b) and cytokeratin (CK)-7 (c); negativity of CK-20 (d) (×400)

After 8 months, the patient returned to our emergency unit due to a decreased level of consciousness. CT scan of the head revealed the presence of multilobulated lesions (Figure 3a) at the very same site where total removal surgery was previously performed. IHC examination demonstrated positive results for TTF-1 and CK-7 but negative result for CK-20 (Figure $3 b-d$ ) which confirms to a diagnosis of lung carcinoma metastasis with $>32 \%$ positivity of moderate intensity to programmed cell death ligand-1. A recent chest radiograph revealed the presence of small $3 \mathrm{~cm}$ nodules in the middle and inferior lobes of the right lung (Figure 4a). A contrast chest CT scan (both coronal and sagittal planes) (Figure $b$ and $c$ ) revealed the presence of a $4.5-\mathrm{cm}$ isohypodense mass that enhanced with contrast in the inferior lobe of the left lung. A fine-needle aspiration biopsy of both lungs was performed and the tumor was finally diagnosed as primary lung ADC with no mutations in the epidermal growth factor receptor (EGFR; using q-RT-PCR for exons 18, 19, 20, and 21; data not shown). However, the patient died 2 weeks after the second surgery due to respiratory failure.
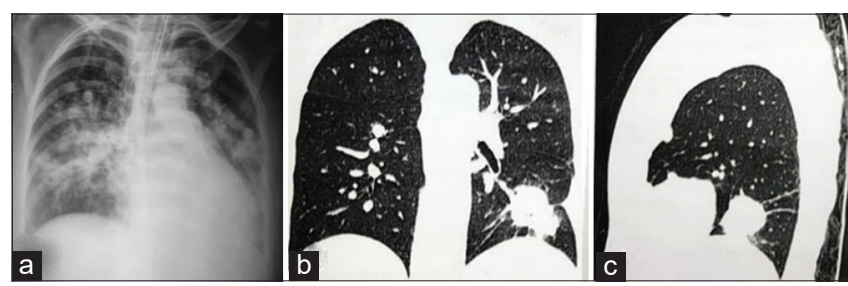

Figure 4: A recent chest radiograph showed the presence of small $3 \mathrm{~cm}$ nodules in the middle and inferior lobe of the right lung (a). A contrast chest computed tomography scan both coronal (b) and sagittal (c) showed the presence of a $4.5 \mathrm{~cm}$ isohypodense mass that enhanced with contrast in the inferior lobe of the left lung

\section{Discussion}

Micropapillary ADC of the lung has been recognized as high-grade variants of lung ADC. The 
diagnosis of micropapillary ADC of the lung confirmed by histopathological examination. Micropapillary is considered equivalent to poorly differentiated ADC and resembles a morphology associated with a worst prognosis [5], [6]. The first report found with our literature query was published in 2002 by Amin et al. [6]; the authors acknowledged that micropapillary carcinoma in lung had been recognized, but no importance had yet been given to it. Miyoshi et al. were the first to describe the morphologic features of the micropapillary pattern in lung primary tumors. They simply described it as "small papillary tufts lacking a central fibrovascular core" [7] which have specific clinical and histopathological characteristics, such as a relatively female predominance and older age, which are consistent with our patient who was a 54-year-old woman. The study reveals that micropapillary ADC of the lung patient showed higher propensity to develop lymph node metastasis and had an overall decreased survival compared to those without a micropapillary component [7], [8], [9], [10], [11], [12].

Cytology became an important tool for diagnosis. Hoshi et al. defined the cytological features important in the diagnosis of micropapillary components as round, three-dimensional, cohesive clusters of neoplastic cells (consisting of $>3$ and $<20$ cells) with a pseudopapillary configuration. In their study, patients with these features in early Stage I disease had worse prognosis compared to controls [13]. Furthermore, a case report by Duncan et al. described the features seen in a primary lung cancer with significant micropapillary components as highly cellular malignant cells arranged in tight three-dimensional clusters, papillae without fibrovascular cores, and discohesive sheets of cells. Their avascular papillary structures and cell clusters were composed of overlapping atypical nuclei with irregular nuclear membranes and prominent nucleoli. In addition, they described many discohesive individual cells and clear spaces at the periphery of the cell balls, accentuated with Romanowsky stain [14]. These features were to diagnosis micropapillary carcinoma at the time and some are still used.

The major diagnostic dilemma arises in the histological diagnosis; in our study, some important IHC examinations were performed. The differential diagnoses include papillary meningioma, which is EMA and CK positive but vimentin negative. In our case, we detected a strongly diffuse CK expression, which suggested a metastatic carcinoma, but somehow, there was no evidence of malignancy in any other part of the body. Other differential diagnoses include papillary ependymoma which is GFAP positive and astroblastoma, which is characterized by a typical palisading pattern of astrocytic cells with broad, nontapering, strongly GFAP-positive processes radiating toward the central blood vessels. GFAP expression is rare; our case revealed GFAP-negative expression in the cell. Tumor invasion can occur into the adjacent brain tissue with abundant blood supply and higher
$\mathrm{Ki}-67$ index; in our case, the malignant features were exhibited with a high Ki-67 index. Due to their rare occurrence, unusual chest radiograph in the first place, and some inconsistencies, the final diagnosis is still dependent on pathological results.

In high-grade variants of lung ADC, such as in our case, chemo- and radio-therapy should be considered at the time of primary diagnosis, regardless of the surgical outcome. However, our patient refused to receive chemoradiotherapy. Eight months later, the patient returned to our emergency unit due to a decreased level of consciousness. CT scan of the head revealed the presence of a multilobulated mass in the very same area where total tumor removal surgery was previously performed. In our case, there were no pre-operative findings indicating the presence of lung cancer. Somehow, the patient refused to receive postoperative chemo-radiotherapy and coincidentally, a multilobulated lesion was found at the very same site as previously operated. Therefore, she was operated for the $2^{\text {nd }}$ time and exhibited an identical histology, which led to a diagnosis of micropapillary ADC of the lung that metastasis to the brain, twice.

In our center, the most common metastasizes tumor to the brain has been found to be lung cancer $(64 \%)$ followed by thyroid (13\%) and breast $(9.4 \%)$ cancer. Several theories have been suggested to explain the mechanism of micropapillary ADC of the lung initially discovered to metastasis into the same site in the brain that has been previously operated twice, including their rich vascularity, originally malignant, fast growth rate that allows short time for metastasis spreading, hormonal factors, and high metabolic rate that provide a competitive metabolic environment. However, the exact mechanism of this phenomenon occurrence remains unclear. Although the primary lesion in the lung seems inhibited, they are activated when they move to the metastatic lesion and entrench themselves in the tissues at the secondary location [15]. This leads to affinity between tumors, which has been speculated to be a cause of same site of metastasis. A high-grade variant of micropapillary ADC of the lung with unusual chest radiograph imaging place and some inconsistencies that may result in a diagnostic dilemma, which can lead to incorrect choices for therapy. In general, brain imaging characteristics might lead to confusion between extra- or intra-axial brain tumors. The mechanism underlying such a lung tumor metastasis is not completely understood and the preoperative diagnosis remains challenging.

\section{Conclusion}

We have reported micropapillary ADC of the lung that metastasized to the brain in the same site, twice, 
even after the first lesion in the brain previously operated totally. Therefore, in our case, a definitive diagnosis was possible only through the histopathological examination along with a good communication between the surgeon and the pathologist.

\section{Consent}

Informed consent was obtained from the patient for publication of this case report and any accompanying images.

\section{References}

1. Wang SY, Ye X, Ou W, Lin YB, Zhang BB, Yang H. Risk of cerebral metastases for postoperative locally advanced nonsmall-cell lung cancer. Lung Cancer. 2009;64(2):238-43. https:// doi.org/10.1016/j.lungcan.2008.08.012

PMid: 18838190

2. Travis WD, Brambilla E, Noguchi M, Nicholson AG, Geisinger KR, Yatabe $\mathrm{Y}$, et al. International association for the study of lung cancer/American thoracic society/European respiratory society international multidisciplinary classification of lung adenocarcinoma. J Thorac Oncol. 2011;6(2):244-85. https://doi.org/10.1513/pats.201107-042st

PMid:21252716

3. Travis WD, Brambilla E, Nicholson AG, Yatabe $Y$, Austin JH, Beasley MB, et al. The 2015 World Health Organization classification of lung tumors: Impact of genetic, clinical and radiologic advances since the 2004 classification. J Thorac Oncol. 2015;10(9):1243-60. https://doi.org/10.341 0/f.725757738.793546406

\section{PMid:26291008}

4. Tsuta K, Kawago M, Inoue E, Yoshida A, Takahashi F, Sakurai H, et al. The utility of the proposed IASLC/ATS/ERS lung adenocarcinoma subtypes for disease prognosis and correlation of driver gene alterations. Lung Cancer. 2013;81(3):371-6. https://doi.org/10.1016/j.lungcan.2013.06.012

PMid:23891509

5. Travis WD. The 2015 WHO classification of lung tumors. Pathologe. 2014;35 Suppl 2:188.

PMid:25394966

6. Amin MB, Tamboli P, Merchant SH, Ordóñez NG, Ro J, Ayala AG, et al. Micropapillary component in lung adenocarcinoma: A distinctive histologic feature with possible prognostic significance. Am J Surg Pathol. 2002;26(3):358-64. https://doi. org/10.1097/00000478-200203000-00010

PMid: 11859208

7. Miyoshi T, Satoh Y, Okumura S, Nakagawa K, Shirakusa T, Tsuchiya E, et al. Early-stage lung adenocarcinomas with a micropapillary pattern, a distinct pathologic marker for a significantly poor prognosis. Am J Surg Pathol. 2003;27(1):101 9. https://doi.org/10.1097/00000478-200301000-00011 PMid:12502932

8. Makimoto $\mathrm{Y}$, Nabeshima K, Iwasaki H, Miyoshi T, Enatsu S, Shiraishi T, et al. Micropapillary pattern: A distinct pathological marker to subclassify tumours with a significantly poor prognosis within small peripheral lung adenocarcinoma $(</=20 \mathrm{~mm})$ with mixed bronchioloalveolar and invasive subtypes (Noguchi's Type C tumours). Histopathology. 2005;46(6):677-84. https:// doi.org/10.1111/j.1365-2559.2005.02126.x PMid:15910599

9. Kamiya K, Hayashi Y, Douguchi J, Hashiguchi A, Yamada T, Izumi $\mathrm{Y}$, et al. Histopathological features and prognostic significance of the micropapillary pattern in lung adenocarcinoma. Mod Pathol. 2008;21(8):992-1001. https://doi. org/10.1038/modpathol.2008.79

PMid: 18516041

10. Kawakami $T$, Nabeshima $K$, Makimoto $Y$, Hamasaki $M$, Iwasaki A, Shirakusa T, et al. Micropapillary pattern and grade of stromal invasion in $\mathrm{pT} 1$ adenocarcinoma of the lung: Usefulness as prognostic factors. Mod Pathol. 2007;20(5):514-21. https:// doi.org/10.1038/modpathol.3800765

PMid: 17334347

11. Roh MS, Lee JI, Choi PJ, Hong YS. Relationship between micropapillary component and micrometastasis in the regional lymph nodes of patients with stage I lung adenocarcinoma. Histopathology. 2004;45(6):580-6. https://doi. org/10.1111/j.1365-2559.2004.01953.x

PMid: 15569048

12. Tsutsumida H, Nomoto M, Goto M, Kitajima S, Kubota I, Hirotsu Y et al. A micropapillary pattern is predictive of a poor prognosis in lung adenocarcinoma, and reduced surfactant apoprotein A expression in the micropapillary pattern is an excellent indicator of a poor prognosis. Mod Pathol. 2007;20(6):638-47. https://doi. org/10.1038/modpathol.3800780

PMid: 17431413

13. Hoshi R, Tsuzuku M, Horai T, Ishikawa Y, Satoh Y. Micropapillary clusters in early-stage lung adenocarcinomas: A distinct cytologic sign of significantly poor prognosis. Cancer. 2004;102(2):81-6. https://doi.org/10.1002/cncr.20125 PMid:15098251

14. Duncan LD, Jacob S, Atkinson S. Fine needle aspiration cytologic findings of micropapillary carcinoma in the lung: A case report. Acta Cytol. 2007;51(4):605-9. https://doi. org/10.1159/000325808 PMid:17718135

15. Moody P, Murtagh K, Piduru S, Brem S, Murtagh R, Rojiani AM Tumor-to-tumor metastasis: Pathology and neuroimaging considerations. Int J Clin Exp Pathol. 2012;5(4):367-73.

PMid:22670183 